\title{
Adaptive Neural Control Based on High Order Integral Chained Differentiator for Morphing Aircraft
}

\author{
Zhonghua Wu, ${ }^{1}$ Jingchao Lu, ${ }^{1}$ Jahanzeb Rajput, ${ }^{1}$ Jingping Shi, ${ }^{1}$ and Wen $\mathrm{Ma}^{2}$ \\ ${ }^{1}$ School of Automation, Northwestern Polytechnical University, Xi'an 710072, China \\ ${ }^{2}$ Science and Technology on Aircraft Control Laboratory, AVIC Xian Flight Automatic Control Research Institute, Xian 710065, China \\ Correspondence should be addressed to Zhonghua Wu; 463897575@qq.com
}

Received 28 July 2015; Accepted 17 September 2015

Academic Editor: Xinguang Zhang

Copyright (c) 2015 Zhonghua Wu et al. This is an open access article distributed under the Creative Commons Attribution License, which permits unrestricted use, distribution, and reproduction in any medium, provided the original work is properly cited.

\begin{abstract}
This paper presents an adaptive neural control for the longitudinal dynamics of a morphing aircraft. Based on the functional decomposition, it is reasonable to decompose the longitudinal dynamics into velocity and altitude subsystems. As for the velocity subsystem, the adaptive control is proposed via dynamic inversion method using neural network. To deal with input constraints, the additional compensation system is employed to help engine recover from input saturation rapidly. The highlight is that high order integral chained differentiator is used to estimate the newly defined variables and an adaptive neural controller is designed for the altitude subsystem where only one neural network is employed to approximate the lumped uncertain nonlinearity. The altitude subsystem controller is considerably simpler than the ones based on backstepping. It is proved using Lyapunov stability theory that the proposed control law can ensure that all the tracking error converges to an arbitrarily small neighborhood around zero. Numerical simulation study demonstrates the effectiveness of the proposed strategy, during the morphing process, in spite of some uncertain system nonlinearity.
\end{abstract}

\section{Introduction}

With the development of morphing wing technology, the flight performance of an aircraft can be improved according to the current flight conditions [1-3]. The morphing aircraft are the flight vehicles that change their shape to either effectuate a change in mission or provide control authority for maneuvering $[4,5]$, without the use of discrete control surfaces or seams. Aircraft with morphing capability exhibit the distinct advantages of being able to fulfill multiple types of missions and to perform extreme maneuvers not possible with conventional aircraft $[6,7]$.

The field of morphing aircraft research is composed of a large array of interdisciplinary studies, including wing structure, actuation systems, aerodynamic modeling, nonrigid dynamics, and flight control [8]. A number of studies have focused on optimization of the actuator locations in the morphing structure units [9-11]. Other relative research work that involves the aeroelastics analysis is presented in [12]. The importance of the inertial forces and moments is studied in [13], with the goal of reducing the dynamics that must be dealt with in the flight control design. A methodology which is suitable for numerical calculation of the dynamic loads for a morphing aircraft is presented in [14]. In [15], linear parameter varying modeling is proposed for a folding wing morphing aircraft during the wing morphing process, whereas the longitudinal dynamic responses are numerically simulated based on the quasi-steady aerodynamic assumption.

Despite significant advances in the development of wing structure, actuation systems, and dynamic model, much work remains to be done to effectively control the morphing aircraft. The control system of a morphing aircraft must be capable of achieving consistent and robust performance meanwhile maintaining stability during large variations in the aircraft geometry, which may severely affect aerodynamic forces, moments of inertia, and center of mass.

For the disturbance rejection, a pair of linear controllers is synthesized for a linear input-varying morphing aircraft in [16]. A simple proportional state feedback control integrated with the eigenstructure assignment is proposed for the span-morphing aircraft in [17]. Based on a linear parameter 
varying model, self-gain scheduled $H_{\infty}$ controller is designed for the wing transition process in [18]. On the basis of varying linear parameter and classical methodology, a synthesized multiloop controller of a morphing unmanned aerial vehicle is formulated to guarantee a good performance subjected to large-scale geometrical shape changes in [19].

To cope with system uncertainties, adaptive control and neural network control techniques have been used for decades. For a linear morphing aircraft dynamic model, an indirect adaptive control method is designed in [6], which comprises the receding horizon optimal control law coupled with the modified sequential least squares parameter identification. In [20], a single network adaptive critic tracking controller design for a morphing aircraft is studied, wherein the set of initial weights of the neural network is determined by using a linear system model, which requires offline pretraining. Based on the concepts of feedback linearization, in [21], a combination of dynamic inversion and structured model reference adaptive control is used for the control of a morphing air vehicle. Typically a morphing aircraft exhibits highly nonlinear dynamics characteristics. Because of the morphing aircraft's design and flight condition, it is extremely sensitive to change in physics as well as aerodynamic parameters. Almost all controller designs discussed above are based on linear models. Moreover the input saturation (physical limitation in engine) has not been considered in any work, which usually appears in many practical systems and severely degrades the closed-loop performance [22].

As a powerful nonlinear technique, backstepping control has been used for control system designs with strict-feedback form, extensively. With conventional backstepping, a possible issue is the explosion of complexity. This is caused by the repeating differentiations of certain nonlinear functions. To efficiently handle the system uncertainty in each subsystem, RBFNN with the universal approximation capability is employed in $[23,24]$. Since RBFNN is used, we need to take derivatives of those radial basis functions, which will further lead to heavier calculation burden in each step design. Recently, the dynamic surface control was employed to solve this problem and many research results were presented $[25,26]$. However, the determination of virtual control terms during the backstepping design requires tedious and complex analysis. More than one neural network is taken for approximation whose complexity increases like the order of the controlled backstepping design.

The motivation of this paper is to present a nonlinear robust adaptive neural controller for the morphing aircraft based on high order integral chained differentiator to achieve stability in the sweeping process where both system uncertainty and input restrictions are considered. The contribution of this paper can be summarized as follows.

Firstly, a nonlinear longitudinal model is derived from a curved-fitted model, with the center of mass position, aerodynamic forces, and the moments of inertia being varied with respect to the morphing parameters. The longitudinal model is then decomposed into altitude and velocity subsystems.

Secondly, the highlight is that the altitude subsystem dynamics is transformed into normal-feedback formulation and a robust adaptive neural controller using HICD is designed where only one neural network is employed to approximate the lumped uncertain system nonlinearity. The controller is considerably simpler than the ones based on backstepping which requires tedious and complex analysis for their virtual control terms. This feature guarantees that the computational burden of the algorithm can be reduced. Moreover the algorithm is convenient for realtime implementation on flight computers. Meanwhile, the adaptive control is proposed for velocity subsystem and an additional compensation system is employed to deal with input constraints, which will help engine recover from input saturation rapidly.

Finally, the Lyapunov synthesis based on stability analysis is used to prove that all the signals in the closed systems are semiglobally uniformly ultimately bounded with tracking error converging to a close neighborhood of origin.

The rest of the paper is organized as follows: Section 2 introduces the model of the morphing aircraft and formulates the normal output-feedback form of the altitude and velocity subsystems of longitudinal dynamics of the morphing aircraft. Section 3 briefly describes the background theory of RBFNN. Section 4 presents the adaptive neural controller design and the stability analysis for altitude and velocity subsystems. The simulation results are presented and discussed in Section 5. Section 6 gives the concluding remarks and future works.

\section{Problem Formulation}

2.1. Morphing Aircraft Model. The control-oriented model of the longitudinal dynamics of a morphing aircraft considered in this study is based on Seigler $[4,5]$. This model comprises five state variables $(V, h, \alpha, \gamma$, and $q)$ and two control inputs $\left(\delta_{e}, T\right)$, where $V$ is the velocity, $h$ is the altitude, $\alpha$ is angle of attack, $\gamma$ is the flight path angle (FPA), and $q$ is the pitch rate; $\delta_{e}$ and $T$ represent elevator deflection and thrust force, respectively. Consider

$$
\begin{aligned}
\dot{V} & =\frac{\left(-D+T \cos \alpha-m g \sin \gamma+F_{I x}\right)}{m}, \\
\dot{h} & =V \sin \gamma, \\
\dot{\gamma} & =\frac{\left[L+T \sin \alpha-m g \cos \gamma-F_{I k z}\right]}{(m V)}, \\
\dot{\alpha} & =\frac{\left[-L-T \sin \alpha+m g \cos \gamma+F_{I z}\right]}{(m V)}+q, \\
\dot{q} & =-\frac{\dot{I}_{y} q}{I_{y}}+\frac{\left(-S_{x} g \cos \theta+M_{A}+T Z_{T}+M_{I y}\right)}{I_{y}}, \\
F_{I x} & =S_{x}\left(\dot{q} \sin \alpha+q^{2} \cos \alpha\right)+2 \dot{S}_{x} q \sin \alpha-\ddot{S}_{x} \cos \alpha, \\
F_{I z} & =F_{I k z} \\
& =S_{x}\left(\dot{q} \cos \alpha-q^{2} \sin \alpha\right)+2 \dot{S}_{x} q \cos \alpha+\ddot{S}_{x} \sin \alpha, \\
M_{I y} & =S_{x}(\dot{V} \sin \alpha+V \dot{\alpha} \cos \alpha-V q \cos \alpha),
\end{aligned}
$$


where $D, L$, and $M_{A}$ represent drag force, lift force, and pitch moment, respectively; $m, I_{y}$, and $g$ denote the mass of aircraft, moment of inertia about pitch axis, and gravity constant; $F_{I x}$, $F_{I z}, F_{I k z}$, and $M_{I y}$ represent inertial force and moment caused by morphing process; $Z_{T}$ is the position of engine in the body axis; $S_{x}$ denotes the static moment distributed in the body axis of $x$; the related definitions are given as follows:

$$
\begin{aligned}
S_{x}(\zeta) \approx & {\left[2 m_{1} r_{1 x}+m_{3} r_{3 x}\right], } \\
Q= & \frac{1}{2 \rho_{h} V^{2}}, \\
L= & C_{L}(\zeta) Q S_{w}(\zeta), \\
D= & C_{D}(\zeta) Q S_{w}(\zeta), \\
M_{A}= & C_{m}(\zeta) Q S_{w}(\zeta) c_{A}(\zeta), \\
C_{L}(\zeta)= & C_{L 0}(\zeta)+C_{L \alpha}(\zeta) \alpha+C_{L \delta e}(\zeta) \delta_{e} \\
\approx & C_{L 0}(\zeta)+C_{L \alpha}(\zeta) \alpha, \\
C_{D}(\zeta)= & C_{D 0}(\zeta)+C_{D \alpha}(\zeta) \alpha+C_{D \alpha 2}(\zeta) \alpha^{2}, \\
C_{m}(\zeta)= & C_{m 0}(\zeta)+C_{m \alpha}(\zeta) \alpha+C_{m \delta e}(\zeta) \delta_{e} \\
& +\frac{C_{m q}(\zeta) q c_{A}(\zeta)}{(2 V)},
\end{aligned}
$$

where $\zeta$ represents the sweep angle, $\rho_{h}$ denotes the air density, $S_{w}$ is the wing surface, $c_{A}$ represents the mean aerodynamic chord, and $b$ is the wingspan. $Q$ and $M_{A}$ denote the dynamic pressure and pitch moment. $C_{L}, C_{D}$, and $C_{m}$ are the total aerodynamic lift force coefficient, drag force coefficient, and pitching moment coefficient, respectively. $m_{1}$ and $m_{3}$ represent the mass of aircraft's wing and body. $r_{1 x}$ and $r_{3 x}$ denote the position of aircraft's wing and body in the aircraftbody coordinate frame.

We assume that the engine model can be expressed as follows [27].

(A) Engine Rate. The dynamics for the engine speed $n$ is modeled by a first-order linear system with the time constant $\tau_{n}$ and the engine speed reference signal $n_{c}$ as follows:

$$
\dot{n}=-\frac{n}{\tau_{n}}+\frac{n_{c}}{\tau_{n}} .
$$

$$
\begin{aligned}
f_{1}\left(x_{1}\right) & =\left(\frac{1}{m V}\right)\left(Q S_{w} C_{L 0}-m g \cos \gamma\right), \\
f_{2}\left(x_{1}, x_{2}\right) & =0, \\
g_{1}\left(x_{1}\right) & =\frac{Q S_{w} C_{L \alpha}}{m V},
\end{aligned}
$$

(B) Thrust Force. The thrust force is generated by the propeller and can be expressed with dimensionless coefficients. The dimensionless thrust coefficient is

$$
C_{F T}(J)=C_{F T 1}+C_{F T 2} J+C_{F T 3} J^{2}
$$

with the ratio $J=V_{T} / D_{T} \pi n$, where the diameter of the propeller is $D_{T}$, the engine speed is $n$, and the airspeed is $V_{T}$. Here we assume that $V_{T}$ is equal to $V$. The thrust force is computed as shown below:

$$
T=\rho_{h} n^{2} D_{T}^{2} C_{F T}(J) .
$$

Remark 1. It is important to point out that $r_{1 x}, r_{3 x}, I_{y}, c_{A}$, $S_{w}, b, C_{L}, C_{D}$, and $C_{m}$ are associated with sweep angle $\zeta$ in the morphing process. Their functional relationships will be shown later in Section 5.

\subsection{System Transformation}

(A) Altitude Subsystem. The tracking error of the altitude is defined as $\widetilde{h}=h-h_{d}$. Furthermore, the altitude command is transformed into the desired flight path angle (FPA). The demand of flight path angle is generated as [22]

$$
\gamma_{d}=\arcsin \left[\frac{\left(-k_{h} \tilde{h}-k_{I} \tilde{h}+\dot{h}_{d}\right)}{V}\right] .
$$

If $k_{h}>0$ and $k_{I}>0$ are chosen appropriately and the FPA is controlled to follow $\gamma_{d}$, then the altitude error is regulated to zero exponentially.

Remark 2. Since the control problem considered in this paper only takes into account cruise trajectories and does not consider the aggressive maneuvering, the thrust $T \sin \alpha$ can be neglected since it is generally much smaller than the lift. In order to transform the altitude subsystem into strict-feedback form, $F_{I k z}$ in (3) is regarded as an unmodeled term.

Define $X=\left[x_{1}, x_{2}, x_{3}\right]^{T}, x_{1}=\gamma, x_{2}=\theta, x_{3}=q, \theta=$ $\alpha+\gamma, u=\delta_{e}$; the strict-feedback forms of equations of the altitude (3)-(5) are rewritten as

$$
\begin{aligned}
& \dot{x}_{1}=f_{1}\left(x_{1}\right)+g_{1}\left(x_{1}\right) x_{2}, \\
& \dot{x}_{2}=f_{2}\left(x_{1}, x_{2}\right)+g_{2}\left(x_{1}, x_{2}\right) x_{3}, \\
& \dot{x}_{3}=f_{3}\left(x_{1}, x_{2}, x_{3}\right)+g_{3}\left(x_{1}, x_{2}, x_{3}\right) u,
\end{aligned}
$$

where 


$$
\begin{aligned}
f_{3}\left(x_{1}, x_{2}, x_{3}\right) & =\frac{\left[Q S_{w} c_{A}\left(C_{m 0}+C_{m \alpha} \alpha+C_{m q} q c_{A} /(2 V)\right)-S_{x} g \cos \theta-\dot{I}_{y} q+T Z_{T}+M_{I y}\right]}{I_{y}}, \\
g_{2}\left(x_{1}, x_{2}\right) & =1, \\
g_{3}\left(x_{1}, x_{2}, x_{3}\right) & =\frac{Q S_{w} c_{A} C_{m \delta e}}{I_{y}} .
\end{aligned}
$$

Assumption 3. $f_{1}, f_{3}, f_{V}, g_{1}, g_{3}$, and $g_{V}$ are unknown smooth functions; we assume that there exist positive constants $\bar{g}_{i 1}$, $\bar{g}_{i 2}, \bar{g}_{V 1}$, and $\bar{g}_{V 2}$ such that $\bar{g}_{i 1} \geq g_{i}(\cdot) \geq \bar{g}_{i 2}, i=1,3$, $\bar{g}_{V 1} \geq g_{V} \geq \bar{g}_{V 2}$. There also exist constants $g_{1 d}$ and $g_{3 d}$ such that $g_{1 d} \geq\left|\dot{g}_{1}\right|, g_{3 d} \geq\left|\dot{g}_{3}\right|$. Meanwhile, in this paper, we assume that all the system states can be measured and there is no time-delay in the signal transmission.

Lemma 4 (high order integral chained differentiator [28]). Suppose the function $\bar{\zeta}(t)$ and its first $n-1$ derivatives are bounded. Consider the following linear system:

$$
\begin{gathered}
\dot{\zeta}_{1}=\varsigma_{2} \\
\dot{\zeta}_{2}=\varsigma_{3} \\
\vdots \\
\dot{\zeta}_{n}=-\frac{a_{f 1}}{\chi^{n}}\left(\varsigma_{1}-\bar{\varsigma}(t)\right)-\frac{a_{f 2}}{\chi^{n-1}} \varsigma_{2} \cdots \frac{a_{f n}}{\chi} \varsigma_{n},
\end{gathered}
$$

where $\chi$ is a small positive constant and parameters $a_{f 1}$ to $a_{f n}$ are chosen such that the polynomial $\bar{s}^{n}+a_{f n} \bar{s}^{n-1}+\cdots+a_{f 2} \bar{s}+$ $a_{f 1}=0$ is Hurwitz. Then

$$
\lim _{\chi \rightarrow 0} \bar{\zeta}_{i}=\varsigma^{(i-1)}(t)
$$

In the following, we show that original system (12) can be transformed into the normal form with respect to the newly defined state variables. Let $z_{1}=x_{1}$ and $z_{2}=\dot{z}_{1}=f_{1}+g_{1} x_{2}$. The derivative of $z_{2}$ with respect to time is formulated as

$$
\begin{aligned}
\dot{z}_{2} & =\frac{\partial f_{1}}{\partial x_{1}} \dot{x}_{1}+\frac{\partial g_{1}}{\partial x_{1}} \dot{x}_{1} x_{2}+g_{1} \dot{x}_{2} \\
& =\left(\frac{\partial f_{1}}{\partial x_{1}}+\frac{\partial g_{1}}{\partial x_{1}} x_{2}\right)\left(f_{1}+g_{1} x_{2}\right)+g_{1} f_{2}+g_{1} g_{2} x_{3} \\
& =a_{2}\left(x_{1}, x_{2}\right)+b_{2}\left(x_{1}, x_{2}\right) x_{3},
\end{aligned}
$$

where $a_{2}\left(x_{1}, x_{2}\right)=\left(\partial f_{1} / \partial x_{1}+\left(\partial g_{1} / \partial x_{1}\right) x_{2}\right)\left(f_{1}+g_{1} x_{2}\right)+g_{1} f_{2}$, $b_{2}\left(x_{1}, x_{2}\right)=g_{1} g_{2}$.
Similarly, let $z_{3}=\dot{z}_{2}=a_{2}+b_{2} x_{3}$ and its time derivative is induced by

$$
\begin{aligned}
\dot{z}_{3} & =\sum_{i=1}^{2} \frac{\partial a_{2}}{\partial x_{i}} \dot{x}_{i}+\sum_{i=1}^{2} \frac{\partial b_{2}}{\partial x_{i}} \dot{x}_{i} x_{3}+b_{2} \dot{x}_{3} \\
& =\sum_{i=1}^{2}\left(\frac{\partial a_{2}}{\partial x_{i}}+\frac{\partial b_{2}}{\partial x_{i}} x_{3}\right)\left(f_{i}+g_{i} x_{i+1}\right)+b_{2}\left(f_{3}+g_{3} u\right) \\
& =a_{3}\left(x_{1}, x_{2}, x_{3}\right)+b_{3}\left(x_{1}, x_{2}, x_{3}\right) u,
\end{aligned}
$$

where $a_{3}=\sum_{i=1}^{2}\left(\partial a_{2} / \partial x_{i}+\left(\partial b_{2} / \partial x_{i}\right) x_{3}\right)\left(f_{i}+g_{i} x_{i+1}\right)+b_{2} f_{3}$ and $b_{3}=g_{1} g_{2} g_{3}$.

As a result, strict-feedback system (12) can be described as the following normal output form with respect to the newly defined state variables $z_{1}, z_{2}$, and $z_{3}$ :

$$
\begin{aligned}
& \dot{z}_{1}=z_{2}, \\
& \dot{z}_{2}=z_{3}, \\
& \dot{z}_{3}=a_{3}+b_{3} u, \\
& y=z_{1}=x_{1} .
\end{aligned}
$$

(B) Velocity Subsystem. With the modeling uncertainties and external disturbance existing, the uncertain nonlinear model can be formulated as

$$
\begin{aligned}
\dot{V} & =\left[f_{V 0}\left(X_{V}\right)+\Delta f_{V}\right]+g_{V}\left(X_{V}\right) T+d_{V} \\
& =f_{V 0}\left(X_{V}\right)+g_{V}\left(X_{V}\right) T+\Delta_{V},
\end{aligned}
$$

where $f_{V}\left(X_{V}\right)=f_{V 0}\left(X_{V}\right)+\Delta f\left(X_{V}\right), g_{V}=(1 / m) \cos \alpha, X_{V}=$ $\left[x_{1}, x_{2}, x_{3}, V\right] . f_{V 0}\left(X_{V}\right)$ is the nominal parts of $f_{V}\left(X_{V}\right) ; \Delta f_{V}$ is the unknown system uncertainties of $f_{V}\left(X_{V}\right) ; d_{V}\left(X_{V}\right)$ is the external disturbance and $\Delta_{V}=\Delta f_{V}\left(X_{V}\right)+d_{V}$ is the lump of system uncertainty.

Remark 5. It should be noted that $a_{3}, b_{3}$ are totally unknown and need to be approached by $\mathrm{NN}$ in the subsequent developments. For the newly defined states $z_{1}, z_{2}$, and $z_{3}$, an HICD will be introduced to estimate them. From Assumption 3, it is also noted that there exist constants $\bar{b}_{3}>0$ and $b_{3 d}>0$ such that $b_{3} \geq \bar{b}_{3}$ and $b_{3 d}>\left|\dot{b}_{3}\right|$. 


\section{Neural Networks}

In many references of robust adaptive control of uncertain nonlinear systems, the RBFNNs are usually employed as approximate model terms for the unknown nonlinear and continuous function terms using their inherent approximation capabilities [25]. As a class of linearly parameterized NNs, RBFNNs are adopted to approximate the unknown and continuous function $H\left(X_{\text {in }}\right): R^{q} \rightarrow R$ which can be written as follows:

$$
H\left(X_{\text {in }}\right)=\widehat{w}^{T} \Phi\left(X_{\text {in }}\right)+\varepsilon,
$$

where $X_{\text {in }} \in R^{q}$ is an input vector of $\mathrm{NN}, \widehat{w} \in R^{p}$ is a weight vector of the NN, $\Phi\left(X_{\text {in }}\right)=\left[\phi_{1}\left(X_{\text {in }}\right), \phi_{1} \cdots \phi_{p}\left(X_{\text {in }}\right)\right]^{T} \in R^{p}$ is a basis function, $\varepsilon$ is the approximation error which satisfies $|\varepsilon| \leq \varepsilon_{\mathrm{re}}$, and $\varepsilon_{\mathrm{re}}$ is a bounded unknown parameter.

In general, an RBFNN can smoothly approximate any continuous function $H\left(X_{\text {in }}\right)$ over the compact $\Omega_{X_{\text {in }}} \in R^{q}$ to any arbitrary accuracy as

$$
H\left(X_{\text {in }}\right)=w^{* T} \Phi\left(X_{\text {in }}\right)+\varepsilon^{*},
$$

where $w^{*}$ is the optimal weight value and $\varepsilon^{*}$ is the smallest approximation error. The Gaussian basis function is written in the form of

$$
\begin{aligned}
& \phi_{i}\left(X_{\mathrm{in}}\right)=\exp \left[-\frac{\left(X_{\mathrm{in}}-c_{i}\right)^{T}\left(X_{\mathrm{in}}-c_{i}\right)}{m_{i}^{2}}\right], \\
& i=1,2, \ldots, p,
\end{aligned}
$$

where $c_{i}$ and $m_{i}$ are the center and width of the neural cell of the $i$ th hidden layer.

Remark 6. There exists an RBFNN in the form of (21) and an optimal parameter vector $w^{*}$ such that $\mid H\left(X_{\text {in }}\right)-$ $w^{* T} \Phi\left(X_{\mathrm{in}}\right)|=| \varepsilon^{*} \mid<\varepsilon_{\mathrm{re}} \cdot \varepsilon_{\mathrm{re}}$ denotes the supremum of the reconstruction error that is inevitably generated. In what follows, the estimation of $w^{*}$ is denoted as $\widehat{w}$.

\section{Control Design and Stability Analysis}

It is easy to note that $h$ is mainly related to $\delta_{e}$ and $V$ is mainly affected by $T$. Therefore, the dynamics can be decoupled into altitude and velocity subsystem and we design the altitude and velocity controller separately. The structure of the proposed control scheme is presented in Figure 1.

4.1. Adaptive Neural Controller for Altitude Subsystem. The control objective of system (12) is to design an adaptive neural controller, which makes $\gamma \rightarrow \gamma_{d}$, and therefore $h \rightarrow h_{d}$, while keeping all the signals involved bounded.
The following controller design is mainly based on the scheme in [29-31]. Vectors $Y_{d}, E$ and a filtered tracking error $s_{\gamma}$ are then defined as follows:

$$
\begin{aligned}
Y_{d} & =\left[y_{d}, \dot{y}_{d}, \ddot{y}_{d}\right]^{T}, \\
E & =Z-Y_{d}, \\
s_{\gamma} & =\left(\frac{d}{d t}+\lambda\right)^{2} E=\left[\begin{array}{ll}
\Lambda^{T} & 1
\end{array}\right] E, \\
e & =y-y_{d}=z_{1}-y_{d},
\end{aligned}
$$

where $Z=\left[\begin{array}{lll}z_{1} & z_{2} & z_{3}\end{array}\right]^{T}, \Lambda=\left[\begin{array}{ll}\lambda^{2} & 2 \lambda\end{array}\right]^{T}$ with $\lambda>0$.

By employing a high order integral chained differentiator, the estimation of $Z=\left[\begin{array}{lll}z_{1} & z_{2} & z_{3}\end{array}\right]^{T}$ is acquired as $\widehat{Z}=$ $\left[\begin{array}{lll}\varsigma_{1} & \varsigma_{2} & \varsigma_{3}\end{array}\right]^{T}$. According to the discussion in [28], there exist positive constant $\varepsilon_{h}$ and $t^{*}$ such that $\forall t>t^{*}$

$$
|\widehat{Z}-Z| \leq \varepsilon_{h} \text {. }
$$

The estimations of $E$ and $s_{\gamma}$ using (14) are denoted as given below:

$$
\begin{aligned}
\widehat{E} & =\widehat{Z}-Y_{d}, \\
\widehat{s}_{\gamma} & =\left[\begin{array}{ll}
\Lambda^{T} & 1
\end{array}\right] \widehat{E} .
\end{aligned}
$$

Based on (25), the derivative of $s_{\gamma}$ with respect to time can be expressed as

$$
\begin{aligned}
\dot{s}_{\gamma} & =\left[\begin{array}{ll}
0 & \Lambda^{T}
\end{array}\right] E+\left(y^{(3)}-y_{d}^{(3)}\right) \\
& =a_{3}+b_{3} u-y_{d}^{(3)}+\left[\begin{array}{ll}
0 & \Lambda^{T}
\end{array}\right] E \\
& =a_{3}+b_{3} u+\widehat{v}-\left[\begin{array}{ll}
0 & \Lambda^{T}
\end{array}\right] \widetilde{E},
\end{aligned}
$$

where $\widehat{v}=-y_{d}^{(3)}+\left[\begin{array}{ll}0 & \Lambda^{T}\end{array}\right] \widehat{E}, \widetilde{E}=\widehat{E}-E=\widehat{Z}-Z$.

Define

$$
u_{a d}^{*}\left(X_{A}, \widehat{v}\right)=\frac{\left(a_{3}+\widehat{v}\right)}{b_{3}} .
$$

$u_{a d}^{*}$ is approximated by RBFNN as

$$
\begin{aligned}
u_{\mathrm{RBF}} & =\widehat{w}_{A}^{T} \Phi\left(X_{A}\right), \\
X_{A} & =\left[X^{T}, \widehat{v}\right],
\end{aligned}
$$

where $\widehat{w}_{A}$ is the estimation of the optimal parameter vector $w_{A}^{*}, \widetilde{w}_{A}=\widehat{w}_{A}-\widehat{w}_{A}^{*}$.

Substituting the unknown $s_{\gamma}$ with $\widehat{s}_{\gamma}$, we determine the control input as follows:

$$
u=-k \widehat{s}_{\gamma}-\widehat{w}_{A}^{T} \Phi\left(X_{A}\right) .
$$

The update law for $\widehat{w}$ is determined as

$$
\begin{aligned}
\dot{\hat{w}}_{A} & =\gamma_{A}\left(\widehat{s}_{\gamma} \Phi\left(X_{A}\right)-\sigma_{s}\left(\widehat{w}_{A}\right) \widehat{w}_{A}\right), \\
\sigma_{s}\left(\widehat{w}_{A}\right) & = \begin{cases}\frac{c_{\Phi}}{\varepsilon_{w}}, & \text { if }\left|\widehat{w}_{A}\right|>\varepsilon_{w} \\
0, & \text { otherwise, }\end{cases}
\end{aligned}
$$




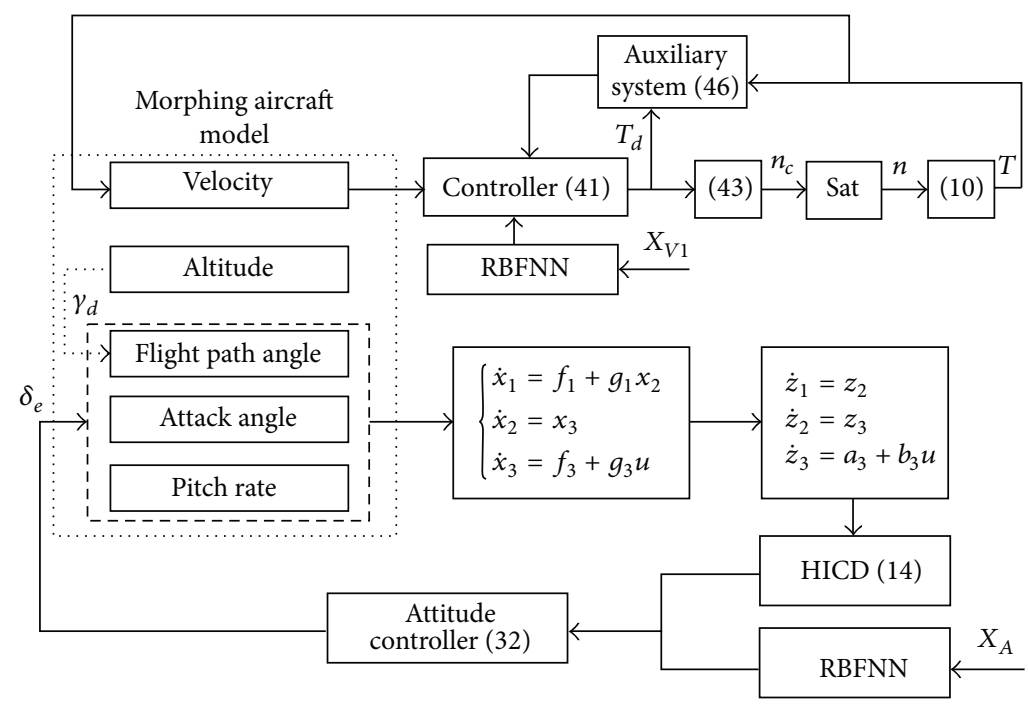

Figure 1: Control scheme.

where $\varepsilon_{W}, c_{\Phi}$ are positive design constants, $\left\|\Phi\left(X_{A}\right)\right\|<c_{\Phi}$, and $\gamma_{A}$ denotes the positive learning rate.

Theorem 7. Consider the adaptive system consisting of (12) under Assumption 3, controller (32) with HICD (14), and adaptive law (33). The filtered error $s_{\gamma}$ and $\widetilde{w}_{A}$ are semiglobally uniformly ultimately bounded.

Proof. Consider the Lyapunov function candidate $L=$ $1 /\left(2 b_{3}\right) s_{\gamma}^{2}+1 /\left(2 \gamma_{A}\right) \widetilde{w}_{A}^{T} \widetilde{w}_{A}$. Taking the time derivation of $L$, we get

$$
\begin{aligned}
& \dot{L}=\frac{s_{\gamma} \dot{s}_{\gamma}}{b_{3}}-\frac{\dot{b}_{3} s_{\gamma}^{2}}{2 b_{3}^{2}}+\frac{\widetilde{w}_{A}^{T} \dot{\hat{w}}_{A}}{\gamma_{A}}=\frac{1}{b_{3}} s_{\gamma}\left(a_{3}+b_{3} u+\widehat{v}\right. \\
& \left.-\left[\begin{array}{ll}
0 & \Lambda^{T}
\end{array}\right] \widetilde{E}\right)-\frac{\dot{b}_{3} s_{\gamma}^{2}}{2 b_{3}^{2}}+\widetilde{w}_{A}^{T}\left(\widehat{s}_{\gamma} \Phi-\sigma_{s}\left(\widehat{w}_{A}\right) \widehat{w}_{A}\right) \\
& =\frac{1}{b_{3}} s_{\gamma}\left(a_{3}+b_{3} u-b_{3} u_{a d}^{*}+b_{3} u_{a d}^{*}+\widehat{v}-\left[\begin{array}{ll}
0 & \Lambda^{T}
\end{array}\right] \widetilde{E}\right) \\
& -\frac{\dot{b}_{3} s_{\gamma}^{2}}{2 b_{3}^{2}}+\widehat{s}_{\gamma} \widetilde{w}_{A}^{T} \Phi-\sigma_{s}\left(\widehat{w}_{A}\right) \widetilde{w}_{A}^{T} \widehat{w}_{A}=\frac{1}{b_{3}} s_{\gamma}\left(-k b_{3} \widehat{s}_{\gamma}\right. \\
& \left.+b_{3}\left(u_{a d}^{*}-\widehat{w}_{A}^{T} \Phi\right)-\left[\begin{array}{ll}
0 & \Lambda^{T}
\end{array}\right] \widetilde{E}\right)-\frac{\dot{b}_{3} s_{\gamma}^{2}}{2 b_{3}^{2}}+s_{\gamma} \widetilde{w}_{A}^{T} \Phi \\
& -\left(s_{\gamma}-\widehat{s}_{\gamma}\right) \widetilde{w}_{A}^{T} \Phi-\sigma_{s}\left(\widehat{w}_{A}\right) \widetilde{w}_{A}^{T} \widehat{w}_{A}=s_{\gamma}\left(-k s_{\gamma}\right. \\
& +k\left(s_{\gamma}-\widehat{s}_{\gamma}\right)+\left(u_{a d}^{*}-w_{A}^{* T} \Phi+w_{A}^{* T} \Phi-\widehat{w}_{A}^{T} \Phi\right) \\
& \left.-\frac{\left[\begin{array}{ll}
0 & \Lambda^{T}
\end{array}\right] \widetilde{E}}{b_{3}}\right)-\frac{\dot{b}_{3} s_{\gamma}^{2}}{2 b_{3}^{2}}+s_{\gamma} \widetilde{w}_{A}^{T} \Phi-\left(s_{\gamma}-\widehat{s}_{\gamma}\right) \widetilde{w}_{A}^{T} \Phi
\end{aligned}
$$

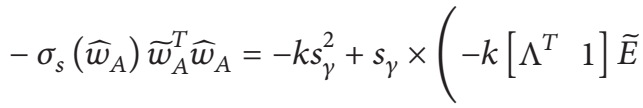

$$
\begin{aligned}
& \left.+\left(u_{a d}^{*}-w_{A}^{* T} \Phi\right)-\widetilde{w}_{A}^{T} \Phi-\frac{\left[\begin{array}{ll}
0 & \Lambda^{T}
\end{array}\right] \widetilde{E}}{b_{3}}\right)-\frac{\dot{b}_{3} s_{\gamma}^{2}}{2 b_{3}^{2}} \\
& +s_{\gamma} \widetilde{w}_{A}^{T} \Phi+\widetilde{w}_{A}^{T}\left[\begin{array}{ll}
\Lambda^{T} & 1
\end{array}\right] \widetilde{E} \Phi-\sigma_{s}\left(\widehat{w}_{A}\right) \widetilde{w}_{A}^{T} \widehat{w}_{A} \\
& \leq-\left(k-\frac{b_{3 d}}{2 \bar{b}_{3}^{2}}\right) s_{\gamma}^{2}+\left|s_{\gamma}\right|\left(k c_{\lambda 1} \varepsilon_{h}+\varepsilon_{\mathrm{re}}+\frac{c_{\lambda 2} \varepsilon_{h}}{\bar{b}_{3}}\right) \\
& +\widetilde{w}_{A}^{T}\left[\begin{array}{ll}
\Lambda^{T} & 1
\end{array}\right] \widetilde{E} \Phi-\sigma_{s}\left(\widehat{w}_{A}\right) \widetilde{w}_{A}^{T} \widehat{w}_{A} .
\end{aligned}
$$

Considering the following facts,

$$
\begin{aligned}
\widetilde{w}_{A}^{T}\left[\begin{array}{ll}
\Lambda^{T} & 1
\end{array}\right] \widetilde{E} \Phi & \leq \frac{1}{8} c_{\Phi} k_{s}\left\|\widetilde{w}_{A}\right\|^{2}+\frac{2}{k_{s}} c_{\Phi}\left\|\left[\begin{array}{ll}
\Lambda^{T} & 1
\end{array}\right] \widetilde{E}\right\|^{2} \\
& =\frac{1}{8} c_{\Phi} k_{s}\left\|\widetilde{w}_{A}\right\|^{2}+\frac{2}{k_{s}} c_{\Phi} \mu_{1}^{2} \\
2 \widetilde{w}_{A}^{T} \widehat{w}_{A} & =\left\|\widetilde{w}_{A}\right\|^{2}+\left\|\widehat{w}_{A}\right\|^{2}-\left\|w_{A}^{*}\right\|^{2} \\
& \geq\left\|\widetilde{w}_{A}\right\|^{2}-\left\|w_{A}^{*}\right\|^{2}
\end{aligned}
$$

we have

$$
\begin{aligned}
\dot{L} \leq & -\left(k-\frac{b_{3 d}}{2 \bar{b}_{3}^{2}}-\frac{1}{2} C_{1}\right) s_{\gamma}^{2} \\
& -\left(\frac{1}{2} \frac{c_{\Phi}}{\varepsilon_{w}}-\frac{1}{8} c_{\Phi} k_{s}\right)\left\|\widetilde{w}_{A}\right\|^{2}+\frac{2}{k_{s}} c_{\Phi} \mu_{1}^{2} \\
& +\frac{1}{2} \frac{c_{\Phi}}{\varepsilon_{w}}\left\|w_{A}^{*}\right\|^{2}+\frac{1}{2} \leq-\rho L+C,
\end{aligned}
$$


where $c_{\lambda 1}=\left\|\left[\begin{array}{ll}\Lambda^{T} & 1\end{array}\right]\right\|, c_{\lambda 2}=\left\|\left[\begin{array}{ll}0 & \Lambda^{T}\end{array}\right]\right\|, \mu_{1}=\left[\begin{array}{ll}\Lambda^{T} & 1\end{array}\right] \widetilde{E}$, $\left|\sigma_{s}(\widehat{w})\right| \leq c_{\Phi} / \varepsilon_{w}, k_{s}>0,\|\Phi\| \leq c_{\Phi},\left|k c_{\lambda 1} \varepsilon_{h}+\varepsilon_{\mathrm{re}}+c_{\lambda 2} \varepsilon_{h} / \bar{b}_{3}\right|=$ $C_{1} . \rho$ and $C$ are given by

$$
\begin{aligned}
& \rho:=\min \left\{\left(k-\frac{b_{3 d}}{2 \bar{b}_{3}^{2}}-\frac{1}{2} C_{1}\right),\left(\frac{1}{2} \frac{c_{\Phi}}{\varepsilon_{w}}-\frac{1}{8} c_{\Phi} k_{s}\right)\right\}, \\
& C:=\frac{2}{k_{s}} c_{\Phi} \mu_{1}^{2}+\frac{1}{2} \frac{c_{\Phi}}{\varepsilon_{w}}\left\|w_{A}^{*}\right\|^{2}+\frac{1}{2} .
\end{aligned}
$$

To ensure the closed-loop stability, the corresponding design parameters should be chosen such that $k-b_{3 d} /\left(2 \bar{b}_{3}^{2}\right)-$ $(1 / 2) C_{1}>0$ and $(1 / 2)\left(c_{\Phi} / \varepsilon_{w}\right)-(1 / 8) c_{\Phi} k_{s}>0$.

According to (37), we have $0 \leq L \leq C / \rho+[L(0)-$ $C / \rho] e^{-\rho t}$. From (37), we can know that $L$ is convergent; that is, $\lim _{x \rightarrow \infty} L=C / \rho$. It can be shown that the filtered signal $s_{\gamma}$ and $\widetilde{w}_{A}$ are semiglobally uniformly bounded.

Remark 8. (1) The switching function $\sigma_{s}\left(\widehat{w}_{A}\right)$ is adopted so that the RBFNN can retain the learned information, which is based on a novel $\sigma$ switching scheme. The adopted switching scheme prevents the loss of information, if $\varepsilon_{w}$ is chosen sufficiently large value such that $\varepsilon_{w}>\left|\widehat{w}_{A}\right|$ while guaranteeing the boundness of $\left|\widehat{w}_{A}\right|$.

(2) It should be noted that, in this paper, only one RBFNN is employed to approximate the lumped uncertain nonlinear function in the altitude subsystem which highlights the simplicity of our proposed controller. However, at least two RBFNNs need to be used in the backstepping scheme, in [25], which require large computational burden. It is also demonstrated that control law and stability analysis is considerably simpler than the previous backstepping-based algorithms.

\subsection{Adaptive Controller for Velocity Subsystem. Define}

$$
\widetilde{V}=V-V_{d} .
$$

Its time derivative is

$$
\dot{\widetilde{V}}=\dot{V}-\dot{V}_{d}=f_{V 0}+g_{V} T+\Delta_{V}-\dot{V}_{d} .
$$

By employing an $\operatorname{RBFNN} \widehat{w}_{V}^{T} \Phi_{V}\left(X_{V 1}\right)$ to approximate unknown uncertainty $\Delta_{V}$, we have

$$
\begin{aligned}
& T_{d} \\
& =\frac{\left[-k_{p V} \widetilde{V}-k_{I V} \int_{0}^{t}\left(\widetilde{V}-V_{e}\right) d \tau-f_{V 0}-\widehat{w}_{V}^{T} \Phi_{V}\left(X_{V 1}\right)+\dot{V}_{d}\right]}{g_{V}},
\end{aligned}
$$

where $X_{V 1}=\left[V, V_{d}, \widetilde{V}\right]$ and $k_{p v}, k_{I v}$ are the positive design parameters; $V_{e}$ is the compensatory term which will be defined as follows. $T_{d}$ represents the desired thrust force.

Equations (9) and (10) are rearranged so as to solve $n_{d}$ in the following equation:

$$
\begin{gathered}
n_{d}^{2}\left(C_{F T 1} \rho_{h} D_{T}^{4}\right)+n_{d}\left(\frac{C_{F T 2} \rho_{h} D_{T}^{3} V}{\pi}\right) \\
+\frac{C_{F T 3} \rho_{h} D_{T}^{2} V^{2}}{\pi^{2}}-T_{d}=0 .
\end{gathered}
$$

In order to solve (42) at each sampling time, $V$ is assumed to be constant during the sampling period. Then

$$
\begin{aligned}
& n_{d}=\frac{\left(c_{n 1} V+\sqrt{c_{n 2} V^{2}+c_{n 3} T_{d}}\right)}{c_{n 4}}, \\
& n_{c}= \begin{cases}n_{\max }, & n_{d} \geq n_{\max } \\
n_{d}, & n_{d} \leq n_{\max },\end{cases}
\end{aligned}
$$

where $c_{n 1}=-C_{F T 2} \rho_{h} D_{T}^{3} / \pi, c_{n 2}=\left(C_{F T 2}^{2}-\right.$ $\left.4 C_{F T 1} C_{F T 3}\right) \rho_{h}^{2} D_{T}^{6} / \pi^{2}, c_{n 3}=4 C_{F T 1} \rho_{h} D_{T}^{4}$, and $c_{n 4}=$ $2 C_{F T 1} \rho_{h} D_{T}^{4}$ are the intermediate variables. $n_{c}$ is the actual engine speed; $n_{\max }$ is the upper limit of $n_{d}$.

Define

$$
\begin{aligned}
\widetilde{V}_{e} & =\widetilde{V}-V_{e}, \\
\dot{V}_{e} & =-k_{p V} V_{e}+g_{V}\left(T-T_{d}\right), \\
V_{e}(0) & =0 .
\end{aligned}
$$

The update law of $\widehat{w}_{V}$ is determined as

$$
\dot{\widehat{w}}_{V}=\eta_{V}\left(\widetilde{V}_{e} \Phi_{V}\left(X_{V 1}\right)-\sigma_{V} \widehat{w}_{V}\right)
$$

where $\sigma_{V}$ is a positive design constant and $\widetilde{w}_{V}=\widehat{w}_{V}-w_{V}^{*} ;(46)$ indicates the auxiliary system used to compensate the engine speed saturation.

The derivatives of $\widetilde{V}$ and $\widetilde{V}_{e}$ with respect to time, $\dot{\bar{V}}$ and $\dot{\vec{V}}_{e}$, can be expressed as

$$
\begin{aligned}
\dot{\vec{V}}= & \dot{V}-\dot{V}_{d}=f_{V 0}+g_{V} T+w_{V}^{* T} \Phi_{V}+\varepsilon_{V}-\dot{V}_{d} \\
= & f_{V 0}+g_{V} T_{d}+g_{V}\left(T-T_{d}\right)+\widehat{w}_{V}^{T} \Phi_{V}-\widetilde{w}_{V}^{T} \Phi_{V} \\
& +\varepsilon_{V}-\dot{V}_{d} \\
= & -k_{p V} \widetilde{V}-k_{I V} \int_{0}^{t} \widetilde{V}_{e} d \tau+g_{V}\left(T-T_{d}\right) \\
& -\widetilde{w}_{V}^{T} \Phi_{V}+\varepsilon_{V}, \\
\dot{\vec{V}}_{e}= & -k_{p V} \widetilde{V}_{e}-k_{I V} \int_{0}^{t}\left(\widetilde{V}_{e}\right) d \tau-\widetilde{w}_{V}^{T} \Phi_{V}+\varepsilon_{V} .
\end{aligned}
$$

Theorem 9. Consider the adaptive system comprising (19), velocity subsystem controller (41) with adaptive law (47), and auxiliary system (46). $\widetilde{V}_{e}$ and $\widetilde{w}_{V}$ are semiglobally uniformly bounded.

Proof. Consider the Lyapunov candidate function

$$
L_{V}(t)=\frac{1}{2} \widetilde{V}_{e}^{2}+\frac{k_{I V}}{2} \int_{0}^{t} \widetilde{V}_{e}^{2} d \tau+\frac{1}{2 \eta_{V}} \widetilde{w}_{V}^{T} \widetilde{w}_{V}
$$


Its time derivative is

$$
\begin{aligned}
\dot{L}_{V}= & \widetilde{V}_{e} \dot{\widetilde{V}}_{e}+k_{I V} \widetilde{V}_{e} \int_{0}^{t} \widetilde{V}_{e} d \tau+\frac{\widetilde{w}_{V}^{T} \dot{\widehat{w}}_{V}}{\eta_{V}} \\
= & \widetilde{V}_{e}\left[-k_{p V} \widetilde{V}_{e}-k_{I V} \int_{0}^{t}\left(\widetilde{V}_{e}\right) d \tau-\widetilde{w}_{V}^{T} \Phi_{V}+\varepsilon_{V}\right] \\
& +k_{I V} \widetilde{V}_{e} \int_{0}^{t} \widetilde{V}_{e} d \tau+\widetilde{w}_{V}^{T}\left(\widetilde{V}_{e} \Phi_{V}-\sigma_{V} \widehat{w}_{V}\right) \\
= & -k_{p V} \widetilde{V}_{e}^{2}+\widetilde{V}_{e} \varepsilon_{V}-\sigma_{V} \widetilde{w}_{V}^{T} \widehat{w}_{V} .
\end{aligned}
$$

Considering the following fact,

$$
\begin{aligned}
2 \widetilde{w}_{V}^{T} \widehat{w}_{V} & =\left\|\widetilde{w}_{V}\right\|^{2}+\left\|\widehat{w}_{V}\right\|^{2}-\left\|w_{V}^{*}\right\|^{2} \geq\left\|\widetilde{w}_{V}\right\|^{2}-\left\|w_{V}^{*}\right\|^{2} \\
\widetilde{V}_{e} \varepsilon_{V} & \leq \frac{1}{2}\left(\widetilde{V}_{e}^{2}+\varepsilon_{V}^{2}\right),
\end{aligned}
$$

we have the following inequality:

$$
\begin{aligned}
\dot{L}_{V} \leq & -\left(k_{p V}-\frac{1}{2}\right) \widetilde{V}_{e}^{2}-\frac{1}{2} \sigma_{V}\left\|\widetilde{w}_{V}\right\|^{2} \\
& +\frac{1}{2}\left(\varepsilon_{V}^{2}+\sigma_{V}\left\|w_{V}^{*}\right\|^{2}\right) \leq-\rho_{V} L_{V}+C_{V},
\end{aligned}
$$

where $\rho_{V}$ and $C_{V}$ are given by $\rho_{V}:=\min \left\{\left(k_{p V}-1 / 2\right), \sigma_{V} / 2\right\}$ and $C_{V}:=\left\{1 / 2 \varepsilon_{V}^{2}+\sigma_{V} / 2\left\|w_{V}^{*}\right\|^{2}\right\}$.

To ensure the closed-loop stability, the corresponding design parameters $k_{p V}, \sigma_{V}$ should be chosen such that $k_{p V}-$ $1 / 2>0, \sigma_{V}>0$. According to (52), it can be shown that the signals $\widetilde{V}_{e}$ and $\widetilde{w}_{V}$ are semiglobally uniformly bounded.

Remark 10. In this section, the dynamic inversion control based on RBFNN is proposed for velocity subsystem with input saturation constraints. To handle the input saturation, auxiliary design system (46) is introduced to analyze the effect of saturation constraint and the auxiliary variable $V_{e}$ is used to design the adaptive law. It is apparent that the constrained control $T$ produced by the designed control command $T_{d}$ can guarantee the closed-loop system's stability.

\section{Numerical Simulation}

In this section, the performance of the developed control strategy applied to the longitudinal model of the morphing aircraft is verified by means of simulations. The aircraft model parameters are shown in Table 1 . Neural network $\widehat{w}_{A}^{T} \Phi\left(X_{A}\right)$ with input vector $X_{A}=\left[x_{1}, x_{2}, x_{3}, \widehat{v}\right]^{T}$ contains 50 nodes with centers $c_{1 i}(i=1 \cdots 50)$ evenly spaced in $\left[-15^{\circ}, 15^{\circ}\right] \times$ $\left[-15^{\circ}, 15^{\circ}\right] \times\left[-15^{\circ}, 15^{\circ}\right] \times\left[-15^{\circ}, 15^{\circ}\right]$ and widths $m_{1 i}(i=$ $1 \cdots 50)=1$; neural network $\widehat{w}_{V}^{T} \Phi\left(X_{V 1}\right)$ with input vector $X_{V 1}=\left[V, V_{d}, \widetilde{V}_{e}\right]^{T}$ contains 10 nodes with centers $c_{2 i}(i=$ $1 \cdots 10)$ evenly spaced in $[10,50] \times[10,50] \times[-50,50]$ and widths $m_{2 i}(i=1 \cdots 10)=5$. The initial condition is set as $X_{0}=\left[\gamma_{0}, \theta_{0}, q_{0}, h_{0}, V_{0}\right]=\left[0,0.99512^{\circ}, 0,1000 \mathrm{~m}, 30 \mathrm{~m} / \mathrm{s}\right]$, $w_{A}(0)=0$, and $w_{V}(0)=0$. Control and HICD parameters are set as $k_{h}=0.5, k_{I}=0.01, k=0.025, \gamma_{A}=0.02, \varepsilon_{w}=10$,
TABLE 1: Morphing aircraft parameters for different configurations.

\begin{tabular}{lccc}
\hline Parameters & $\zeta=0^{\circ}$ & $\zeta=30^{\circ}$ & $\zeta=45^{\circ}$ \\
\hline$S /\left(\mathrm{m}^{2}\right) v$ & 1.6040 & 1.168 & 0.958 \\
$c_{A} /(\mathrm{m})$ & 0.4874 & 0.411 & 0.416 \\
$b /(\mathrm{m})$ & 3.3494 & 2.981 & 2.503 \\
$I_{y} /(\mathrm{kg} \cdot \mathrm{m})$ & 6.4929 & 7.882 & 8.606 \\
\hline
\end{tabular}

$c_{\Phi}=20$, and $\lambda=5 ; k_{p v}=5, k_{I v}=10, \eta_{V}=10$, and $\sigma_{V}=0.01$; $a_{f 1}=10, a_{f 2}=10, a_{f 3}=10$, and $\chi=0.04$. Reference commands are smoothened via several second-order filters shown in (53) below. The engine speed saturation $n_{\max }$ which is set at $4900 \mathrm{RPM}$ is deliberately tightened to explore the capability of the designed controller in adhering to the limits. Consider

$$
\begin{aligned}
\frac{h_{d}}{h_{d 0}} & =\frac{0.64}{s^{2}+1.6 s+0.64}, \\
\frac{V_{d}}{V_{d 0}} & =\frac{1}{s^{2}+2 s+1}, \\
\frac{\zeta_{d}}{\zeta_{d 0}} & =\frac{1}{s^{2}+4 s+4} .
\end{aligned}
$$

Choosing $\zeta=0^{\circ}, 5^{\circ}, \ldots, 45^{\circ}$ as the 10 reference points, the longitudinal aerodynamic parameters for different variation configurations can be computed through computational fluid dynamics (CFD). Then the aerodynamic parameters of the morphing aircraft during wing-transforming process can be linearly interpolated by those of static configurations with the help of MATLAB:

$$
\begin{aligned}
C_{L 0}= & 0.0042 \zeta^{3}-0.1374 \zeta^{2}-0.0516 \zeta+0.2291, \\
c_{A}= & 0.2054 \zeta^{2}-0.2520 \zeta+0.4874 \\
C_{L \alpha}= & -1.1264 \zeta^{3}-0.4351 \zeta^{2}+0.3816+4.592 \\
b= & -1.4599 \zeta^{2}+0.0644 \zeta+3.3494 \\
C_{D 0}= & -0.0024 \zeta^{3}+0.0045 \zeta^{2}+0.0022 \zeta+0.021, \\
C_{D \alpha}= & -0.0310 \zeta^{2}-0.0458 \zeta+0.109 \\
C_{D \alpha 2}= & -1.2990 \zeta^{4}+1.8282 \zeta^{3}-0.7039 \zeta^{2}-0.0258 \zeta \\
& +1.097 \\
S= & -0.8271 \zeta+1.6040 \\
C_{m \alpha}= & 9.6542 \zeta^{3}-6.5395 \zeta^{2}-6.1887 \zeta-1.5909 \\
C_{m 0}= & 0.4239 \zeta^{2}-0.4462 \zeta^{2}-0.0365 \\
C_{m \delta e}= & -0.1624 \zeta^{2}-0.9376 \zeta-0.7889 \\
I_{y}= & -4.9021 \zeta^{3}+6.5774 \zeta^{2}+0.5500 \zeta+6.4929 \\
C_{m q}= & 41.4537 \zeta^{3}-50.4868 \zeta^{2}-9.7741 \zeta-10.673
\end{aligned}
$$



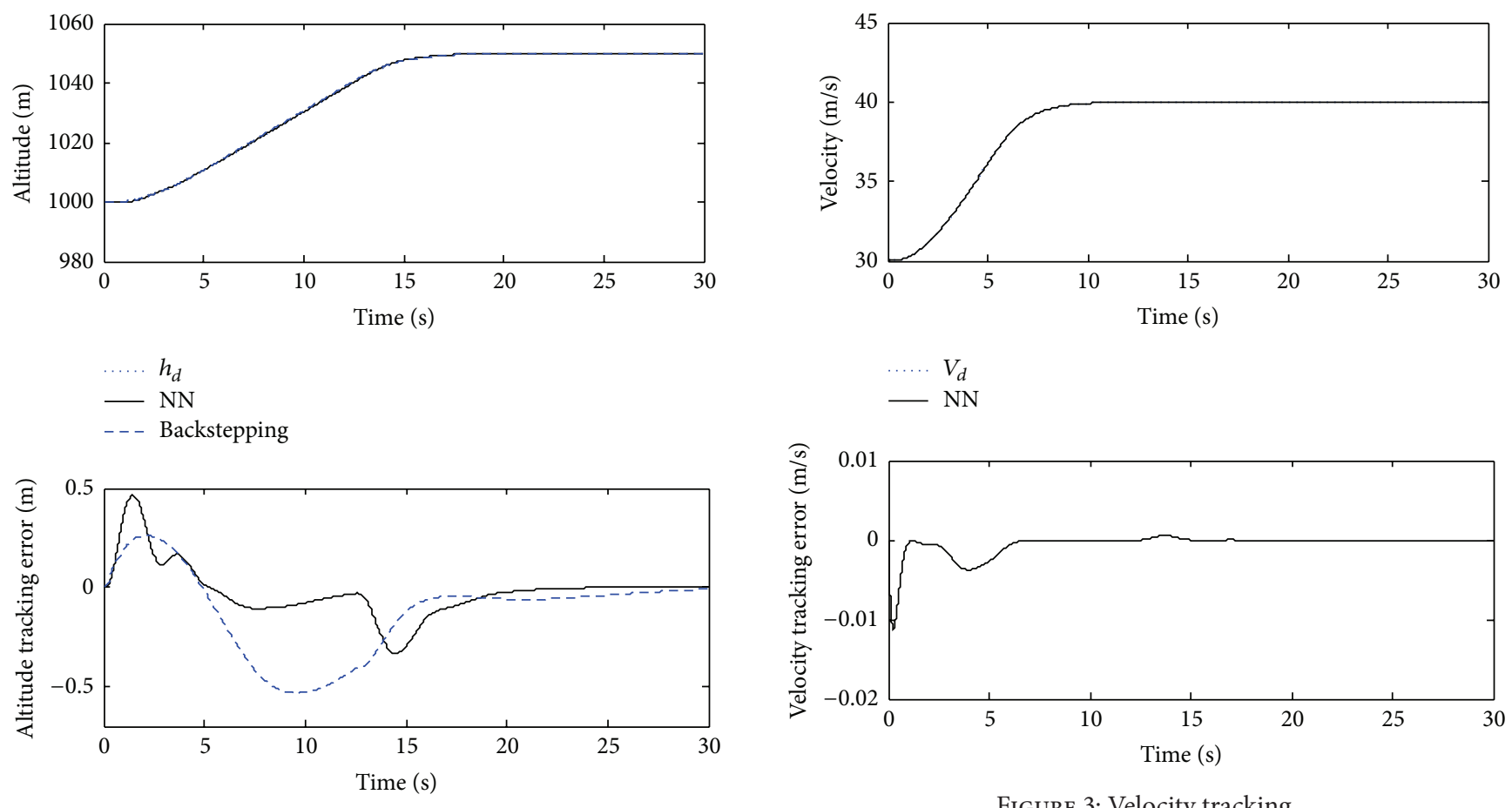

FIgURE 3: Velocity tracking.

$-\mathrm{NN}$
--- Backstepping

FIGURE 2: Altitude tracking.

Due to the complex nonlinear aerodynamic of the morphing aircraft, the aerodynamics is not modeled precisely, the same as it appears in the actual flight conditions. Thus it is significant for the controller to have the ability to provide stability in spite of modeling errors due to unmodeled dynamics and plant parameter variations. To demonstrate the robustness of the proposed control scheme, $20 \%$ aerodynamic uncertainties are taken into account. The following two scenario simulations are employed to test the performance of the proposed controller in handling with aerodynamic uncertainty and input constraints compared with backstepping controller designed in the altitude subsystem.

Scenario 1 . (A) The altitude $h_{d}$ and velocity $V_{d}$ reference commands are generated to make the aircraft climb from $1000 \mathrm{~m}$ to $1050 \mathrm{~m}$ and accelerate from $30 \mathrm{~m} / \mathrm{s}$ to $40 \mathrm{~m} / \mathrm{s}$ in $20 \mathrm{~s}$, where the engine speed saturation is not considered. The simulation results of the tracking output are shown in Figures 2 and 3 ("NN" denotes the simulation results based on adaptive NN controller in this paper and "backstepping" represents the backstepping method in [25]). It can be observed that the system outputs $h$ and $V$ on the basis of NN and backstepping follow the desired trajectory of $h_{d}$ and $V_{d}$ well. The altitude tracking error of NN is smaller than the one based on backstepping. These simulation results show that good tracking performance can be obtained under the proposed adaptive NN control.

(B) To illustrate the effectiveness of the proposed adaptive $\mathrm{NN}$ control further, the sweep reference signal taking place

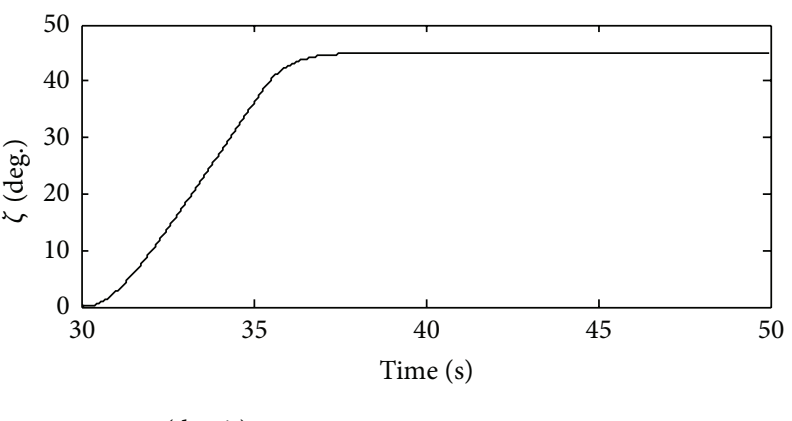

$-9(\operatorname{deg} . / \mathrm{s})$

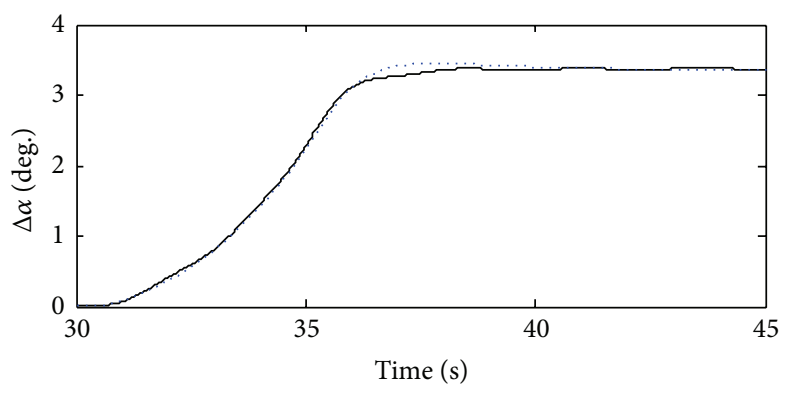

$\begin{array}{ll}\text { - } & \text { NN } \\ \ldots . . . & \text { Backstepping }\end{array}$

FIGURE 4: Sweep signal and angle of attack.

at $30 \mathrm{~s}$ is generated to make the aircraft sweep from $0^{\circ}$ to $45^{\circ}$ at the rate of $9^{\circ} / \mathrm{s}$. The simulation results are shown in Figures $4-7$. It is clear that the velocity is almost constant, during the sweeping process, and the altitude which decreases about $0.32 \mathrm{~m}$ based on adaptive $\mathrm{NN}$ which is better than backstepping method decreases about $1.75 \mathrm{~m}$. They can both 

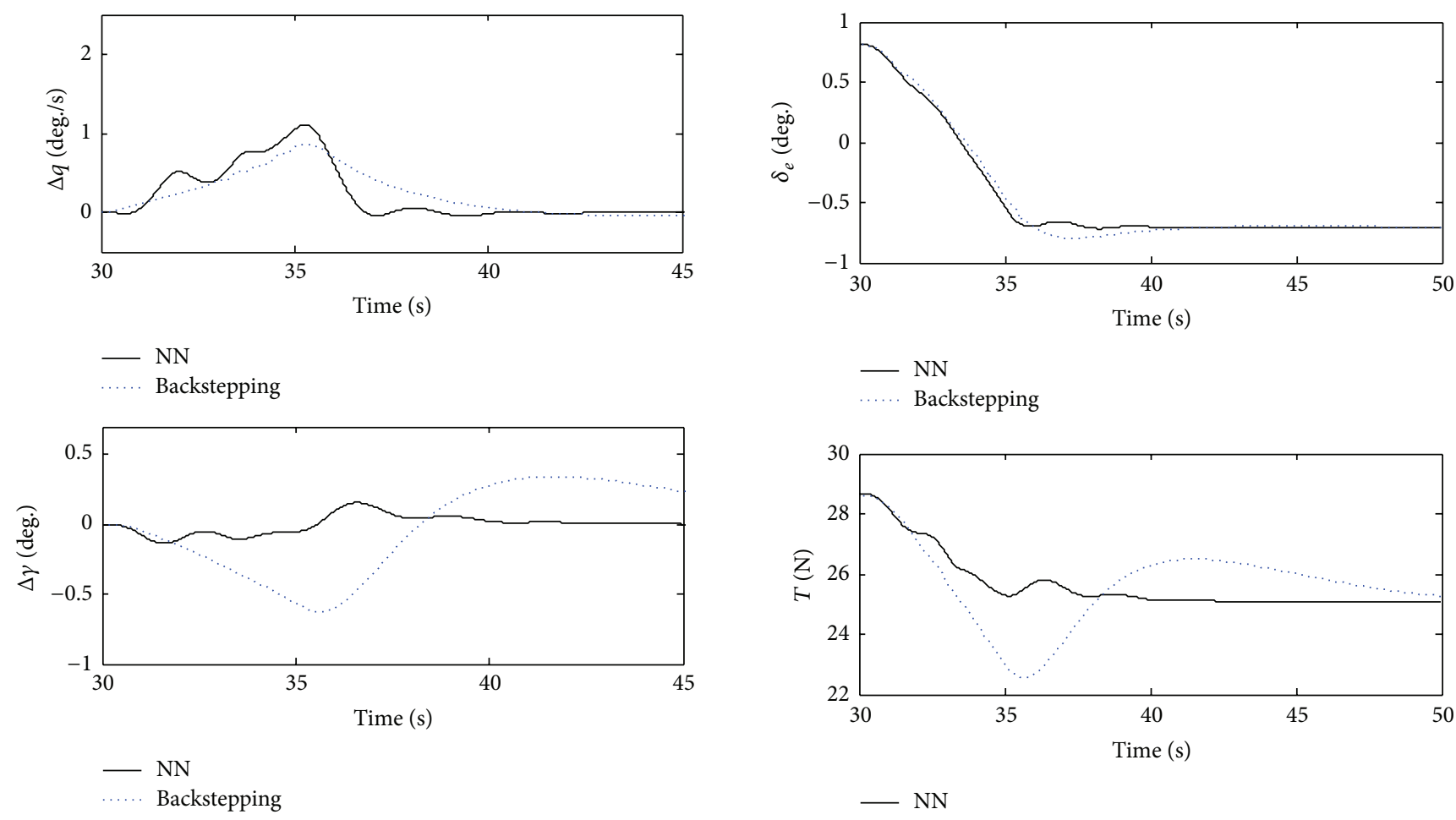

FIGURE 5: Pitch rate and FPA.
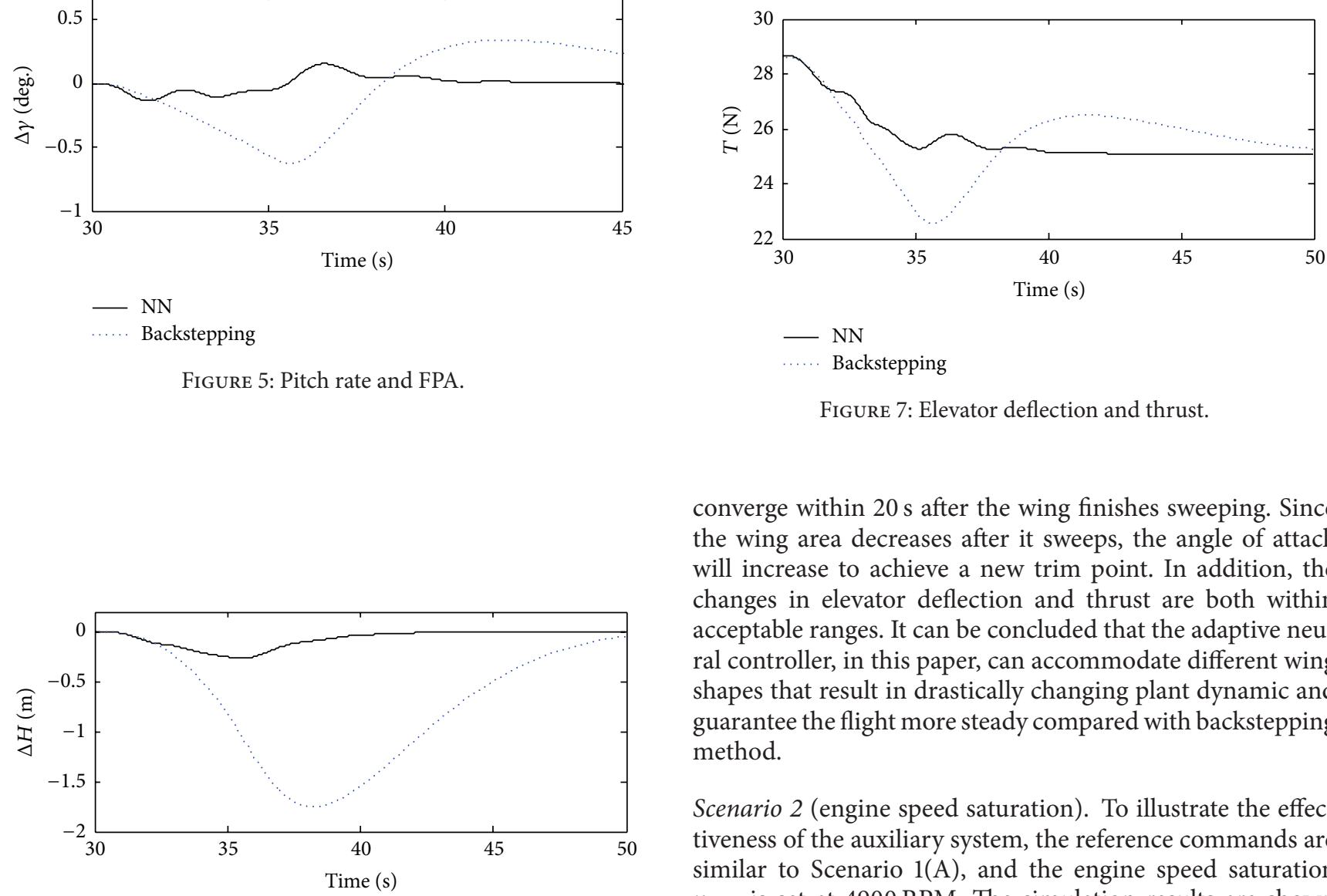

FIgURE 7: Elevator deflection and thrust.

converge within $20 \mathrm{~s}$ after the wing finishes sweeping. Since the wing area decreases after it sweeps, the angle of attack will increase to achieve a new trim point. In addition, the changes in elevator deflection and thrust are both within acceptable ranges. It can be concluded that the adaptive neural controller, in this paper, can accommodate different wing shapes that result in drastically changing plant dynamic and guarantee the flight more steady compared with backstepping method.

Scenario 2 (engine speed saturation). To illustrate the effectiveness of the auxiliary system, the reference commands are similar to Scenario 1(A), and the engine speed saturation $n_{\max }$ is set at $4900 \mathrm{RPM}$. The simulation results are shown in Figures 8 and 9. Due to engine speed saturation, it is obvious to observe that the velocity tracking errors are different between the used ( $V_{1}$ with $0.5 \mathrm{~m} / \mathrm{s}$ to the maximum) and unused ( $V_{2}$ with $0.8 \mathrm{~m} / \mathrm{s}$ to the maximum) additional system. As shown in Figure 9, the engine speed recovers from saturation in $9 \mathrm{~s}$ for $V_{1}$ which is better than $V_{2}$ which recovers in $15 \mathrm{~s}$. These simulation results show that good tracking performance can be obtained under the proposed additional system.

\section{Conclusions and Future Works}

A robust adaptive neural controller based on high order integral chained differentiator is developed for the nonlinear longitudinal model of a morphing aircraft, where aerodynamic uncertainty and engine input constraint are taken into 

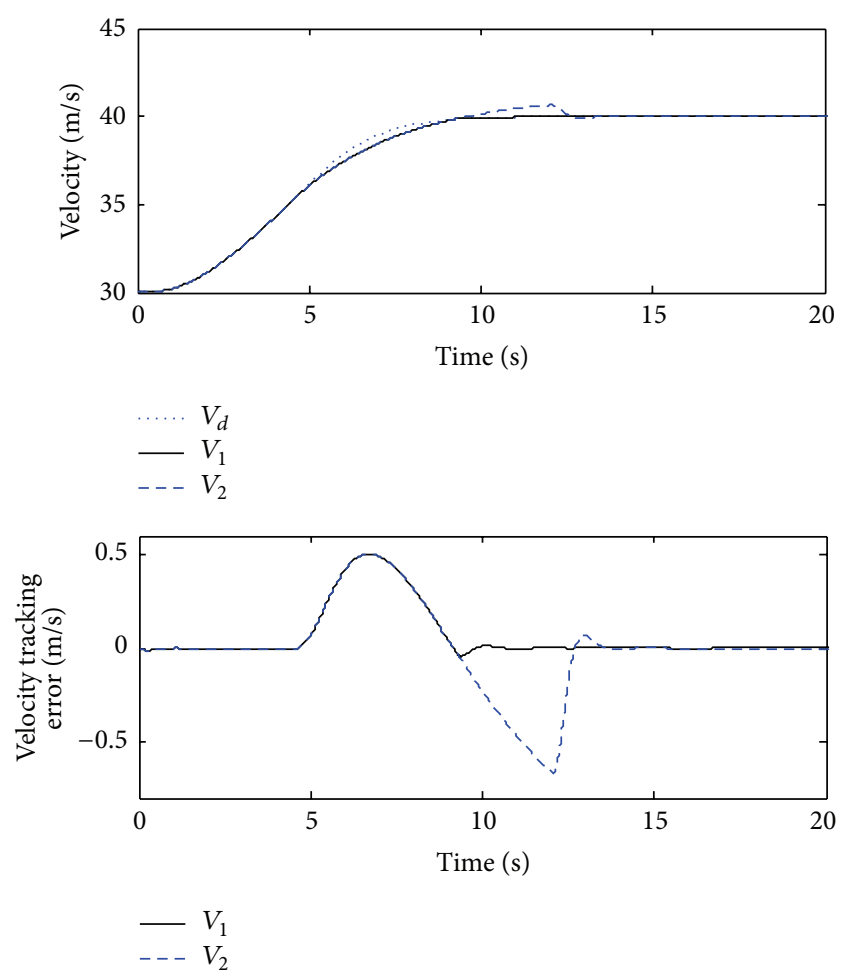

FIGURE 8: Velocity tracking error with input saturation.

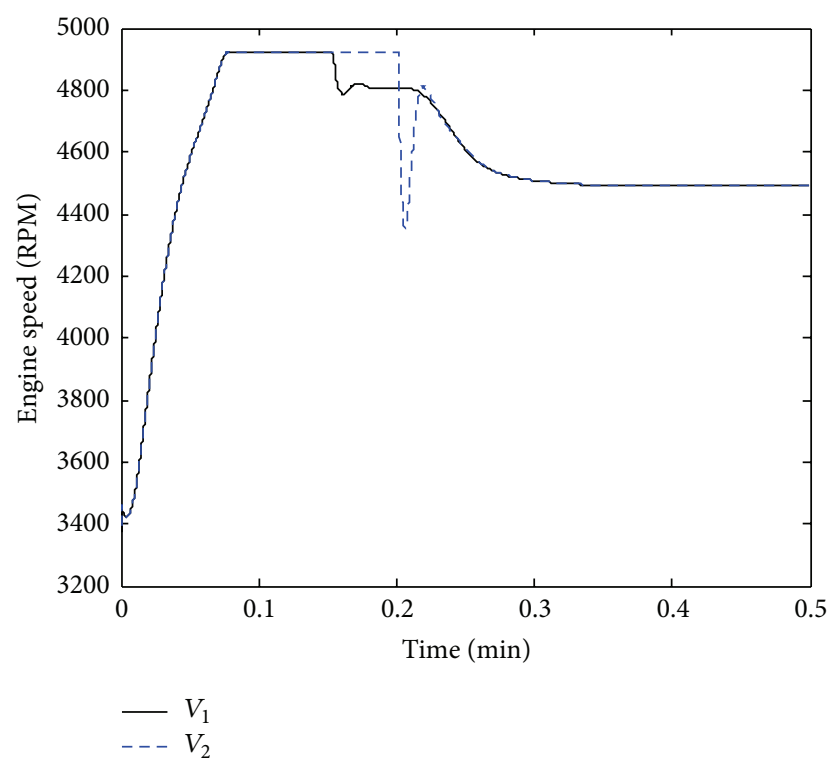

FIGURE 9: Engine speed.

consideration. The altitude controller is viewed as the outputfeedback control problem with one NN to approximate the lumped uncertain nonlinearity while another adaptive NN controller is designed for the velocity subsystem. The problem posed by engine input constraints is overcome by additional systems. The filtered tracking error is proved to be guaranteed zero semiglobally and all the signals are uniformly bounded.
The performance of the presented method is verified by simulations, from which we can deduce that the good performance has been ensured.

For future work, we will analyze how minimal parameter learning technique can be implemented on morphing aircraft in order to reduce the computation burden further. Also it is important to do research on theoretical analysis deeply for the system with input nonlinearity and time-delay where it is still an open problem for this scheme.

\section{Conflict of Interests}

The authors declare that there is no conflict of interests regarding the publication of this paper.

\section{Acknowledgments}

The authors would like to express their sincere thanks to anonymous reviewers for their helpful suggestions for improving the technique note. This work is partially supported by the Natural Science Foundation of China (Grant no. 61374032) and Aeronautical Science Foundation of China (Grant no. 20140753012).

\section{References}

[1] J. Bowman, B. Sanders, B. Cannon, J. Kudva, and S. Joshi, "Development of next generation morphing aircraft structures," in Proceedings of the 48th AIAA/ASME/ASCE/AHS/ASC Structures, Structural Dynamics, and Materials Conference, Honolulu, Hawaii, USA, April 2007.

[2] A. R. Rodriguez, "Morphing aircraft technology survey," in Proceedings of the 5th AIAA Aerospace Sciences Meeting and Exhibit, Reno, Nev, USA, January 2007.

[3] L. Yuping and H. Zhen, "A survey of morphing aircraft control systems," Acta Aeronautica et Astronautica Sinica, vol. 30, no. 10, pp. 1906-1911, 2009.

[4] T. M. Seigler, Dynamics and control of morphing aircraft [Ph.D. thesis], 2005.

[5] T. M. Seigler, D. A. Neal, J.-S. Bae, and D. J. Inman, "Modeling and flight control of large-scale morphing aircraft," Journal of Aircraft, vol. 44, no. 4, pp. 1077-1087, 2007.

[6] N. Gandhi, A. Jha, J. Monaco, T. Seigler, D. Ward, and D. Inman, "Intelligent control of a morphing aircraft," in Proceedings of the 46th AIAA/ASME/ASCE/AHS/ASC Structures, Structural Dynamics and Materials Conference, Honolulu, Hawaii, USA, April 2007.

[7] S. Barbarino, O. Bilgen, R. M. Ajaj, M. I. Friswell, and D. J. Inman, "A review of morphing aircraft," Journal of Intelligent Material Systems and Structures, vol. 22, no. 9, pp. 823-877, 2011.

[8] R. Shi and W. Wan, "Analysis of flight dynamics for largescale morphing aircraft," Aircraft Engineering and Aerospace Technology, vol. 87, no. 1, pp. 38-44, 2015.

[9] B. O'Grady, Multi-objective optimization of a three cell morphing wing substructure [Ph.D. thesis], University of Dayton, Dayton, Ohio, USA, 2010.

[10] T. Johnson, M. Frecker, M. Abdalla, Z. Gurdal, and D. Lindner, "Nonlinear analysis and optimization of diamond cell morphing wings," Journal of Intelligent Material Systems and Structures, vol. 20, no. 7, pp. 815-824, 2009. 
[11] J. J. Joo and B. Sanders, "Optimal location of distributed actuators within an in-plane multi-cell morphing mechanism," Journal of Intelligent Material Systems and Structures, vol. 20, no. 4, pp. 481-492, 2009.

[12] G. R. Andersen, D. L. Cowan, and D. J. Piatak, "Aeroelastic modeling, analysis and testing of a morphing wing structure," in Proceedings of the 48th AIAA/ASME/ASCE/AHS/ASC Structures, Structural Dynamics, and Materials Conference, pp. 359-373, Honolulu, Hawaii, USA, April 2007.

[13] T. M. Seigler, D. A. Neal, and D. J. Inman, "Dynamic modeling of large-scale morphing aircraft," in Proceedings of the 47th AIAA/ASME/ASCE/AHS/ASC Structures, Structural Dynamics and Materials Conference, pp. 3668-3678, Newport, RI, USA, May 2006.

[14] B. Obradovic and K. Subbarao, "Modeling of flight dynamics of morphing-wing aircraft," Journal of Aircraft, vol. 48, no. 2, pp. 391-402, 2011.

[15] T. Yue, L. Wang, and J. Ai, "Longitudinal linear parameter varying modeling and simulation of morphing aircraft," Journal of Aircraft, vol. 50, no. 6, pp. 1673-1681, 2013.

[16] K. Boothe, K. Fitzpatrick, and R. Lind, "Controllers for disturbance rejection for a linear input-varying class of morphing aircraft," in Proceedings of the 46th AIAA/ASME/ASCE/AHS/ASC Structures, Structural Dynamics \& Materials Conference, Austin, Tex, USA, April 2005.

[17] S. Beaverstock, R. A. M. Friswell, W. Dettmer, R. de Breuker, and N. Werter, "Effect of span-morphing on the longitudinal flight stability and control," in Proceedings of the AIAA Guidance, Navigation, and Control Conference, Boston, Mass, USA, August 2013.

[18] T. Yue, L. Wang, and J. Ai, "Gain self-scheduled $H_{\infty}$ control for morphing aircraft in the wing transition process based on an LPV model," Chinese Journal of Aeronautics, vol. 26, no. 4, pp. 909-917, 2013.

[19] D. H. Baldelli, D.-H. Lee, R. S. Sánchez Peñal, and B. Cannon, "Modeling and control of an aeroelastic morphing vehicle," Journal of Guidance, Control, and Dynamics, vol. 31, no. 6, pp. 1687-1699, 2008.

[20] W. G. Nobleheart, S. L. Geethalakshmi, A. Chakravarthy, and J. Steck, "Single Network Adaptive Critic (SNAC) architecture for optimal tracking control of a morphing aircraft during a pullup maneuver," in Proceedings of the AIAA Guidance, Navigation, and Control (GNC) Conference, Boston Mass, USA, August 2013.

[21] J. Valasek, A. Lampton, and M. Marwaha, "Morphing unmanned air vehicle intelligent shape and flight control," in Proceedings of the AIAA Infotech@ Aerospace Conference and AIAA Unmanned... Unlimited Conference, Seattle, Wash, USA, April 2009.

[22] Q. Zong, F. Wang, B. Tian, and R. Su, "Robust adaptive dynamic surface control design for a flexible air-breathing hypersonic vehicle with input constraints and uncertainty," Nonlinear Dynamics, vol. 78, no. 1, pp. 289-315, 2014.

[23] W. Chenliang and L. Yan, "Adaptive dynamic surface control for linear multivariable systems," Automatica, vol. 46, no. 10, pp. 1703-1711, 2010.

[24] Y.-J. Liu, C. L. P. Chen, G.-X. Wen, and S. Tong, "Adaptive neural output feedback tracking control for a class of uncertain discrete-time nonlinear systems," IEEE Transactions on Neural Networks, vol. 22, no. 7, pp. 1162-1167, 2011.

[25] M. Chen, G. Tao, and B. Jiang, "Dynamic surface control using neural networks for a class of uncertain nonlinear systems with input saturation," IEEE Transactions on Neural Networks and Learning Systems, vol. 26, no. 9, pp. 2086-2097, 2014.

[26] S. Luo, S. Wu, Z. Liu, and H. Guan, "Wheeled mobile robot RBFNN dynamic surface control based on disturbance observer," ISRN Applied Mathematics, vol. 2014, Article ID 634936, 9 pages, 2014.

[27] G. J. Ducard, Fault-tolerant Flight Control and Guidance Systems: Practical Methods for Small Unmanned Aerial Vehicles, Springer, 2009.

[28] J. Liu and X. Wang, Advanced Sliding Mode Control for Mechanical Systems, Springer, 2012.

[29] B. Xu, D. X. Gao, and S. X. Wang, "Adaptive neural control based on HGO for hypersonic flight vehicles," Science China Information Sciences, vol. 54, no. 3, pp. 511-520, 2011.

[30] J.-H. Park, S.-H. Kim, and C.-J. Moon, "Adaptive neural control for strict-feedback nonlinear systems without backstepping," IEEE Transactions on Neural Networks, vol. 20, no. 7, pp. 12041209, 2009.

[31] S. N. Huang, K. K. Tan, and T. H. Lee, "Further results on adaptive control for a class of nonlinear systems using neural networks," IEEE Transactions on Neural Networks, vol. 14, no. 3, pp. 719-721, 2003. 


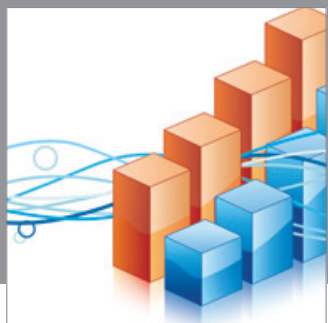

Advances in

Operations Research

mansans

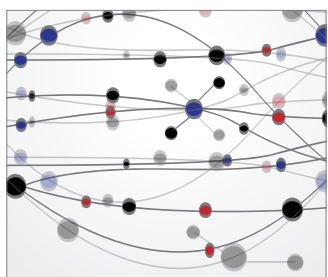

The Scientific World Journal
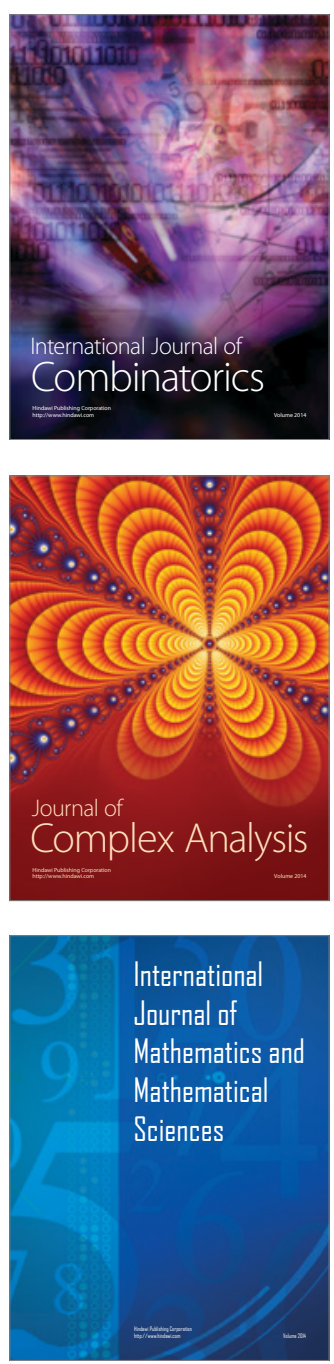
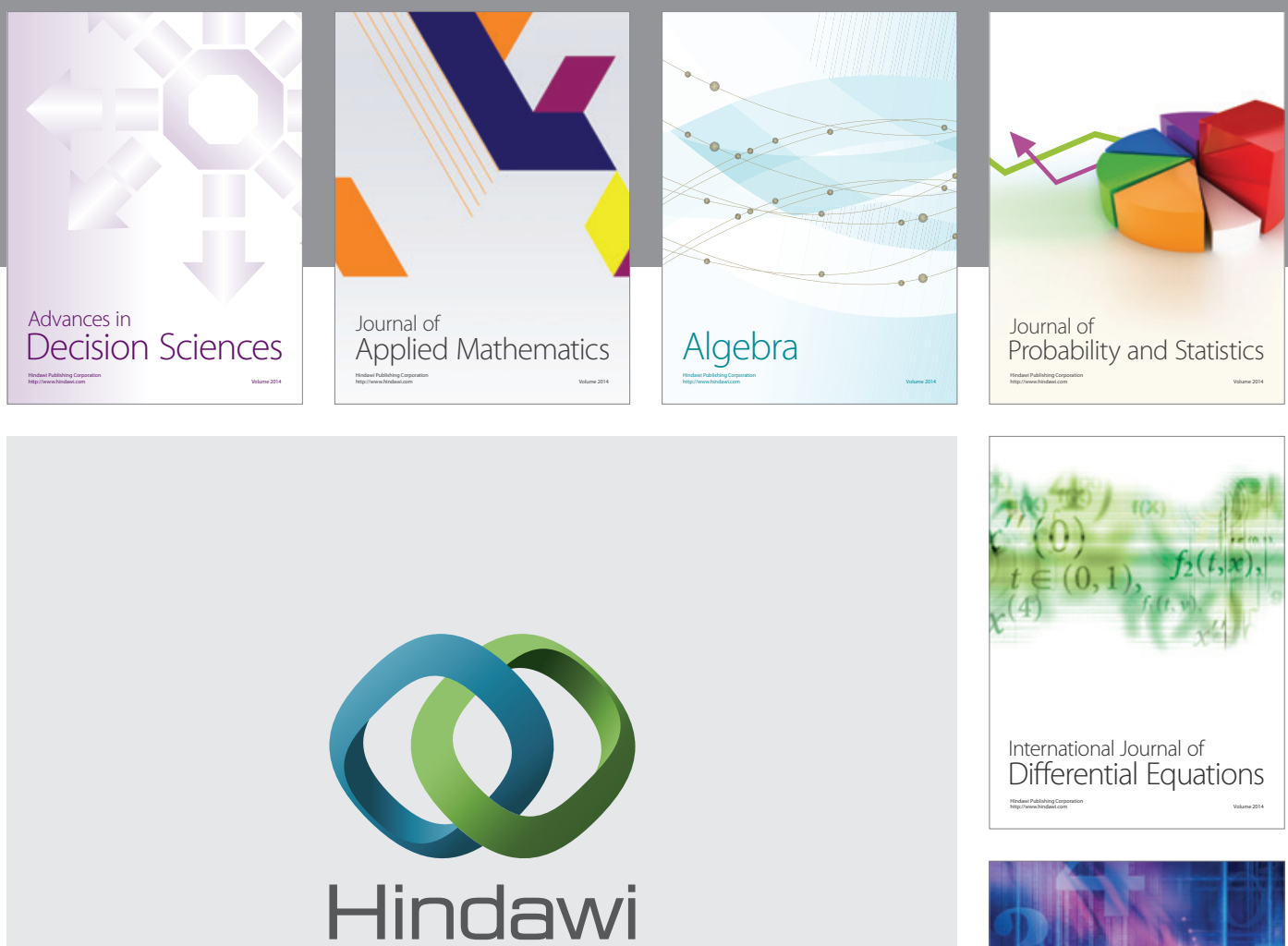

Submit your manuscripts at http://www.hindawi.com
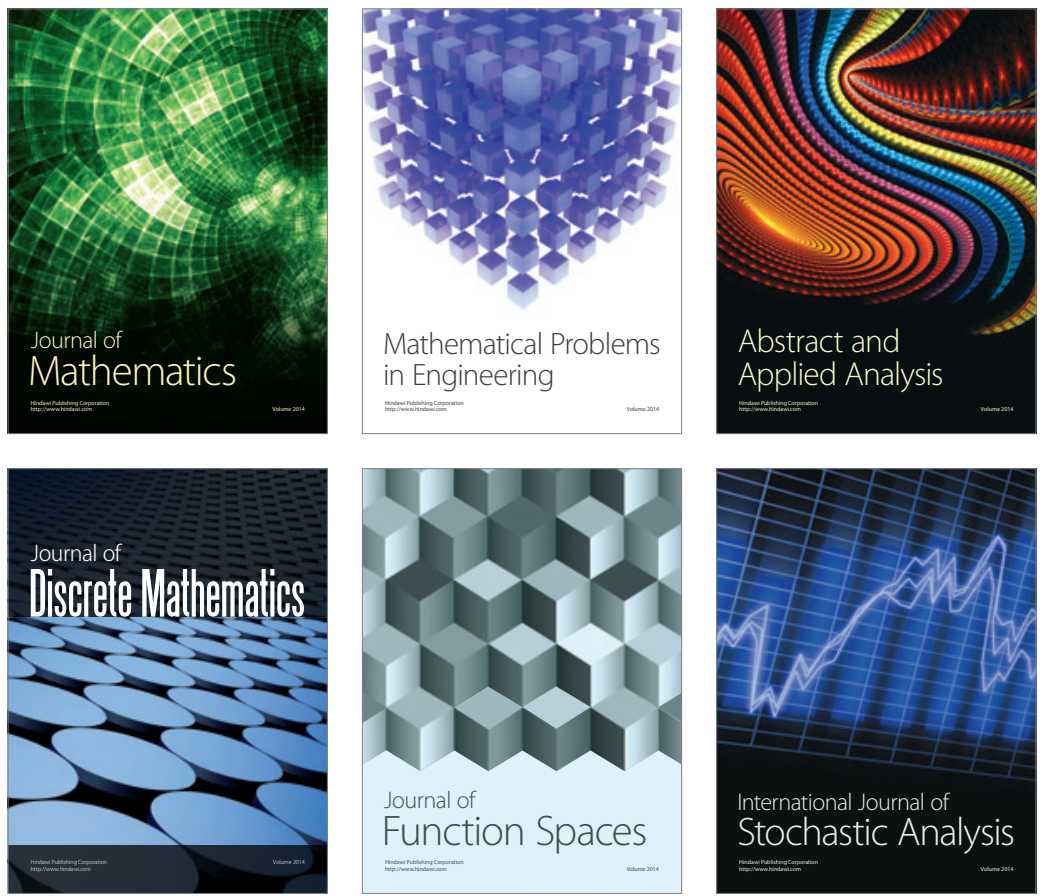

Journal of

Function Spaces

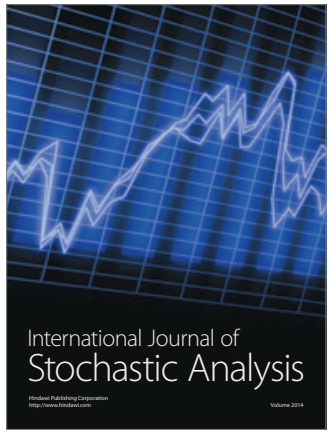

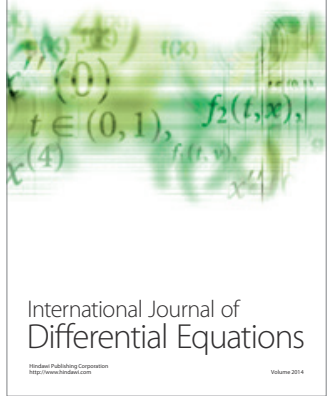
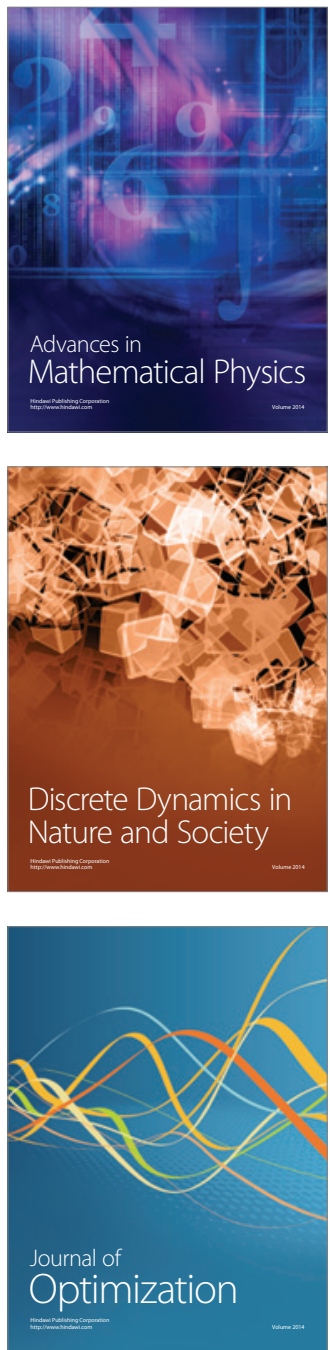